\title{
A healthy Nordic diet and physical performance in old age: findings from the longitudinal Helsinki Birth Cohort Study
}

\author{
Mia-Maria Perälä1,2*, Mikaela von Bonsdorff ${ }^{1}$, Satu Männistö ${ }^{1}$, Minna K. Salonen ${ }^{1,2}$, Mika Simonen ${ }^{4}$, \\ Noora Kanerva ${ }^{1}$, Pertti Pohjolainen ${ }^{5}$, Eero Kajantie ${ }^{1,6,7}$, Taina Rantanen ${ }^{3}$ and Johan G. Eriksson ${ }^{1,2,8,9}$ \\ ${ }^{1}$ Department of Health, National Institute for Health and Welfare, FI-00271 Helsinki, Finland \\ ${ }^{2}$ Folkhälsan Research Center, FI-OO251 Helsinki, Finland \\ ${ }^{3}$ Department of Health Sciences, Gerontology Research Center, University of Jyvaskyla, FI-40014 Jyväskylä, Finland \\ ${ }^{4}$ Department of Social Research, University of Helsinki, FI-OOO14 Helsinki, Finland \\ ${ }^{5}$ Age Institute, FI-O0520 Helsinki, Finland \\ ${ }^{6}$ Hospital for Children and Adolescents, Helsinki University Central Hospital and University of Helsinki, FI-OOO29 Helsinki, Finland \\ ${ }^{7}$ Department of Obstetrics and Gynaecology, Medical Research Center Oulu, Oulu University Hospital and University of Oulu, \\ FI-90014 Oulu, Finland \\ ${ }^{8}$ Department of General Practice and Primary Health Care, University of Helsinki and Helsinki University Hospital, Unit of \\ General Practice, FI-OOO14 Helsinki, Finland \\ ${ }^{9}$ Vaasa Central Hospital, FI-65130 Vaasa, Finland
}

(Submitted 20 July 2015 - Final revision received 2 December 2015 - Accepted 8 December 2015)

\section{Abstract}

Epidemiological studies have shown that a number of nutrients are associated with better physical performance. However, little is still known about the role of the whole diet, particularly a healthy Nordic diet, in relation to physical performance. Therefore, we examined whether a healthy Nordic diet was associated with measures of physical performance 10 years later. We studied 1072 participants from the Helsinki Birth Cohort Study. Participants' diet was assessed using a validated 128-item FFQ at the mean age of 61 years, and $a$ priori-defined Nordic diet score (NDS) was calculated. The score included Nordic fruits and berries, vegetables, cereals, PUFA:SFA and trans-fatty acids ratio, low-fat milk, fish, red and processed meat, total fat and alcohol. At the mean age of 71 years, participants' physical performance was measured using the Senior Fitness Test (SFT), and an overall SFT score was calculated. Women in the highest fourth of the NDS had on average 5 points higher SFT score compared with those in the lowest fourth $\left(P_{\text {for trend }} 0.005\right)$. No such association was observed in men. Women with the highest score had $17 \%$ better result in the 6 -min walk test, $16 \%$ better arm curl and $20 \%$ better chair stand results compared with those with the lowest score (all $P$ values $<0 \cdot 01$ ). In conclusion, a healthy Nordic diet was associated with better overall physical performance among women and might help decrease the risk of disability in old age.

Key words: Physical performance: Senior Fitness Test: Nordic diet: Ageing

Physical performance is an important indicator of current health status of ageing individuals. Poor physical performance in old age has serious health consequences as it predisposes to disability, morbidity as well as mortality ${ }^{(1-4)}$. Reduction in physical performance can also lead to loss of independence and increase the need for healthcare and social care ${ }^{(5-7)}$. As the number of people over 65 years is growing rapidly worldwide ${ }^{(8)}$, it is important to understand lifestyle factors that are related to physical performance, and thus enhance older adults' independence and prevent adverse health outcomes ${ }^{(9)}$.

There is strong evidence that physical activity is an important modifiable factor associated with physical performance, whereas evidence on the effect of diet is more limited.
Some observational studies have investigated the associations between single nutrients and physical performance and have reported positive associations with vitamin $\mathrm{D}^{(10-12)}$, antioxidants $^{(13,14)}$ and $n-3$ fatty acids ${ }^{(15)}$. Relatively little is known about the whole diet and overall physical performance. The few existing studies have shown that people who adhere to the Mediterranean diet have better physical performance ${ }^{(16)}$ and a slower decline of mobility when ageing ${ }^{(17,18)}$. In addition, it has been observed that adherence to overall nutrition recommendation is related to better physical performance in old age ${ }^{(19)}$. Most of these studies have assessed physical functioning with questionnaires or tested only selected aspects of physical performance, such as measuring lower-body

Abbreviations: NDS, Nordic diet score; SFT, Senior Fitness Test.

* Corresponding author: M.-M. Perälä, email mia.perala@thl.fi 
strength using the Short Physical Performance Battery (SPPB). However, it is important to measure all key physiological parameters that are needed for performing normal everyday activities, and thus focus on overall physical functioning.

Although previous studies have shown that the Mediterranean diet is associated with better physical performance, adherence to the Mediterranean diet may be hard to implement globally because of differences in food culture and resources. Therefore, diets that take into account regional food culture and resources are needed. For example, a healthy Nordic diet illustrates the healthier choices of the diet consumed in the Nordic countries and is rich in foods available in the Nordic countries, including apples and berries, roots and cabbages, rye, oats and barley, lowfat milk products, rapeseed oil and fish (salmon and Baltic herring); it is also low in red meat, processed meat products and alcohol $^{(20,21)}$. It has been reported that a healthy Nordic diet contains lower amounts of meat products and sweets and higher amounts of plant food and fish than a diet of Swedish reference population $^{(20)}$. Studies have shown a positive effect of a healthy Nordic diet on several CVD risk factors such as blood pressure ${ }^{(22)}$ and inflammation ${ }^{(23)}$ as well as all-cause mortality ${ }^{(24)}$. Whether a healthy Nordic diet also predicts overall physical performance in old age is still unknown. Therefore, the purpose of this study was to expand our current understanding of the association between diet and overall physical performance and investigate whether a Nordic diet, which illustrates healthier dietary choices based on foods that are typically grown in the Nordic countries, is related to physical performance 10 years later in older men and women.

\section{Methods}

\section{Design and study population}

The subjects of this study belong to the Helsinki Birth Cohort Study, which includes 4630 men and 4130 women who were born as singletons at the Helsinki University Central Hospital between 1934 and 1944, attended child welfare clinics in the city and lived in Finland in $1971^{(25)}$. We used random-number tables to select a subset of people who attended a clinical examination during 2001-2004. Of the 2902 invited subjects, 2003 men ( $n$ 928) and women ( $n$ 1075) participated in the examination $^{(26)}$. In 2011-2013, 1404 participants (627 men and 777 women) were still traceable and were invited to a follow-up clinical examination: 151 had died, 212 had declined participation in subsequent follow-up evaluations and 236 lived abroad or further than $100 \mathrm{~km}$ from Helsinki. A total of 1094 participants ( 478 men and 616 women) attended the clinical examination between 2011 and 2013. Of these, 1072 had adequate information on physical performance tests and food intake and were included in this study.

Those who participated in a follow-up clinical examination were younger at baseline (61.3 v. 61.9 years), had lower BMI $\left(27.2 v \cdot 28 \cdot 2 \mathrm{~kg} / \mathrm{m}^{2}\right)$, had a university degree $(23.9 v .18 \cdot 1 \%)$ and were less likely to smoke at baseline (19.2 v. 29.2\%) compared with those who did not participate $\left(P_{\text {for all }}<0.05\right)$. In addition, their energy intake was lower $(9.2 v .9 .6 \mathrm{MJ})$ and the Nordic diet score (NDS) was higher $(13.0 v .12 .4)\left(P_{\text {for all }}<0.05\right)$. Fat, protein, carbohydrate and alcohol intakes were similar in both groups.
Both studies were conducted according to the guidelines laid down in the Declaration of Helsinki, and all the procedures involving human subjects were approved by the Coordinating Ethics Committee of the Hospital District of Helsinki and Uusimaa. Written informed consent was obtained from all the participants.

\section{Dietary assessment}

At the baseline clinical examination, dietary intake information was collected using a validated, self-administered, 128-item FFQ designed to assess average food intake over the previous 12 months ${ }^{(27,28)}$. For each food item and mixed dish, participants indicated their average frequency of consumption over the previous year by checking one of nine frequency categories ranging from 'never or seldom' to 'at least six times a day'. At the clinic, participants completed the FFQ, which was then checked by a research nurse. The final decision regarding the completeness of the FFQ was made by a nutritionist. Exclusions were made because of incompletely filled FFQ and daily energy intake cut-off points corresponding to $0.5 \%$ at both ends of the daily energy intake distributions for men and women. The average daily intakes of nutrients and foods were calculated using the national food composition database Fineli ${ }^{\circledR(29)}$.

We used a priori-defined NDS, which is also known as the Baltic Sea Diet Score, as a measure of a healthy Nordic diet ${ }^{(21)}$. In brief, the NDS is based on nine components. Favourable food components including fruits and berries (apples, pears and berries), vegetables (tomatoes, lettuce, cucumbers, cabbages, legumes and roots - potatoes excluded), cereals (rye, oats and barley), low-fat milk (fat-free milk and milk with fat content under $2 \%$ ), fish (salmon, Baltic herring) and a ratio of PUFA:SFA and trans-fatty acids were assigned values of $0-3$ according to ascending sex-specific quartile ranks. Unfavourable food components including red meat and meat products (beef, pork, processed meat products) and intake of total fat expressed as a percentage of total energy intake were assigned values of $0-3$ according to descending sex-specific quartile ranks. In addition, men consuming $20 \mathrm{~g}$ or less and women consuming $10 \mathrm{~g}$ or less of alcohol per day received 1 point, or else 0 point was assigned. The NDS ranged from 0 to 25 , where a higher score indicates greater adherence to the healthy Nordic diet.

\section{Physical performance}

At the follow-up clinical examination, physical performance was assessed using the validated Senior Fitness Test (SFT) battery ${ }^{(30,31)}$. We used a modified test battery that consisted of five measurements of physical fitness: (1) number of chair stands during $30 \mathrm{~s}$ to assess lower-body strength, (2) arm curl to assess upper-body strength (curling weight, $2 \mathrm{~kg}$ for women and $3 \mathrm{~kg}$ for men, through the full range of motion as many times as possible during $30 \mathrm{~s}$ ), (3) chair sit and reach to assess lower-body (hamstring) flexibility (from sitting position at front of the chair, distance between fingers and toe), (4) back scratch to assess upper-body (shoulder) flexibility (with one hand reaching over the shoulder and one up the middle of the back, distance between extended middle fingers) and (5) 6-min walk 
test to measure aerobic endurance (distance walked in $6 \mathrm{~min}$ ). All the measurements were performed by a team of trained research assistants.

For each test, the scores of the participant were classified with respect to percentile tables of normative data for each 5 -year age group ${ }^{(31)}$. A rating from 1 to 20 was given according to each 5-percentile range, with 1 being the worst performance (score below the 5 th percentile), 2 the score from the 5 th to the 9th percentile and 20 the best performance (in or above the 95th percentile). We then established the overall score, which was the sum of the normalised scores at the five SFT tests. The overall SFT score varied between 5 and 100 .

\section{Covariates}

Age, BMI, educational status, physical activity, smoking status and total energy intake were all considered as potential confounders of the association between the NDS and physical capacity. During the baseline clinical examination, height was measured to the nearest $0.1 \mathrm{~cm}$ and weight to $0.1 \mathrm{~kg}$. BMI was calculated as weight in kilograms divided by the square of height in metres. Energy intake was measured using an FFQ as an average intake (in $\mathrm{MJ} / \mathrm{d}$ ). On the basis of the questionnaires, the level of educational attainment was classified as elementary school, vocational school, senior high school and university degree. For smoking status, participants were categorised into never-smokers, former smokers and current smokers. Participants' physical activity was assessed using a questionnaire. At the baseline clinical examination, participants completed a validated exercise questionnaire (Kuopio Ischaemic Heart Disease Risk Factor Study (KIHD) 12-month leisure-time physical activity (LTPA) history) $)^{(32)}$. This questionnaire was used to assess duration, frequency and intensity of the most common forms of LTPA, including indoor and outdoor activities performed during the previous 12 months. For each intensity grade, activity-specific metabolic equivalent values were used. Total exercise includes various types of leisuretime activities, from house cleaning and gardening to, for example, jogging and resistance training, during leisure time.

\section{Statistical methods}

The relationship between the NDS and physical performance was examined visually and with a linear regression analysis. We tested for interactions between the NDS and sex on overall SFT score $(P=0.01)$. As the interaction was significant, women and men were analysed separately. Models were adjusted for possible confounding factors: model 1 was adjusted for age and model 2 for age, BMI, educational attainment, physical activity, smoking status and total energy intake. In addition, we analysed associations between the NDS and SFT components and associations of each NDS component with overall SFT score, adjusting for the same potential confounders. All the statistical analyses were carried out using SPSS Statistics version 22 for Windows ${ }^{\circledR}$ (SPSS Inc.); significance level was set at $P<0 \cdot 05$.

\section{Results}

The final analysis included 600 women and 472 men. Tables 1 and 2 show the baseline characteristics of women and men according to quartiles of the NDS. Women with the highest score for the NDS were on average slightly older, more educated, more physically active and less likely to be current smokers than those with the lowest NDS. Men with higher NDS were more physically active and less often current smokers compared with those with a low score (all $P$ values $<0 \cdot 01$ ). Men and women with higher NDS had higher intake of total energy, carbohydrates and fibre and lower intakes of total fat, SFA and MUFA compared with those with a low score (all $P$ values $<0.001$ ). Mean intakes of $\beta$-carotenes, folate and vitamins $\mathrm{B}_{6}, \mathrm{C}, \mathrm{D}$ and $\mathrm{E}$ differed significantly across the NDS quartiles, such that higher intakes were associated with a higher NDS (all $P$ values $<0 \cdot 001$ ).

We observed a statistically significant association between the NDS and the overall SFT score in women (Table 3). In a fully adjusted model, the overall SFT score was 0.55 (95\% CI $0 \cdot 22,0 \cdot 88$ ) points higher per 1 unit increase in the NDS. Women in the highest fourth of the NDS had on average 5 points higher overall SFT score compared with the lowest fourth of the NDS ( $P_{\text {for trend }}$ 0.005). Among men, the NDS was not associated statistically significantly with the overall SFT score. Regarding single SFT components, in a fully adjusted model with the NDS as a continuous variable, 1 point higher NDS was associated with $0 \cdot 16(95 \% \mathrm{CI} 0 \cdot 06,0 \cdot 26)$ unit higher 6 -min walk test score, 0.18 (95\% CI 0.09, 0.28) unit higher arm curl and 0.11 (95\% CI $0 \cdot 03,0 \cdot 19)$ unit higher chair stand test results. The 6 -min walk test score was on average $17 \%$ higher among those women with the highest NDS compared with those with the lowest score $\left(P_{\text {for trend }}\right.$ 0.002). A similar statistically significant association was observed in men after adjusting for age. However, in a fully adjusted model, the association was no longer statistically significant in men. In addition, women with the highest NDS had $16 \%$ better arm curl test results and $20 \%$ better chair stand test results compared with those with the lowest score (all $P$ values $<0 \cdot 01)$. There were no significant associations between the NDS and upper-body (shoulder) flexibility or lower-body (hamstring) flexibility either in women or in men (Table 3). LTPA was not statistically significantly associated with SFT after adjustment for age, BMI, educational attainment and smoking status in women $(P=0 \cdot 087)$ or in men $(P=0 \cdot 12)$.

To better understand the individual role of each NDS component, we examined the associations between each NDS component and the overall SFT score (Table 4). In women, high consumption of fruit and berries, cereals and alcohol were positively related to the overall SFT score, whereas intake of total fat and consumption of red and processed meat were inversely associated with the SFT score. In men, high consumption of cereals and low consumption of low-fat milk were significantly associated with better overall SFT score.

\section{Discussion}

In this study of ageing women and men, we found that among women a higher adherence to a healthy Nordic diet at baseline was associated with better physical performance 10 years later, even after controlling for physical activity. A healthy Nordic diet was especially associated with better results in the 6-min walk, 
Table 1. Lifestyle factors and nutrient intakes according to the Nordic diet score (NDS) quartiles in women ( $n$ 600) at baseline (Mean values with their standard errors or proportions)

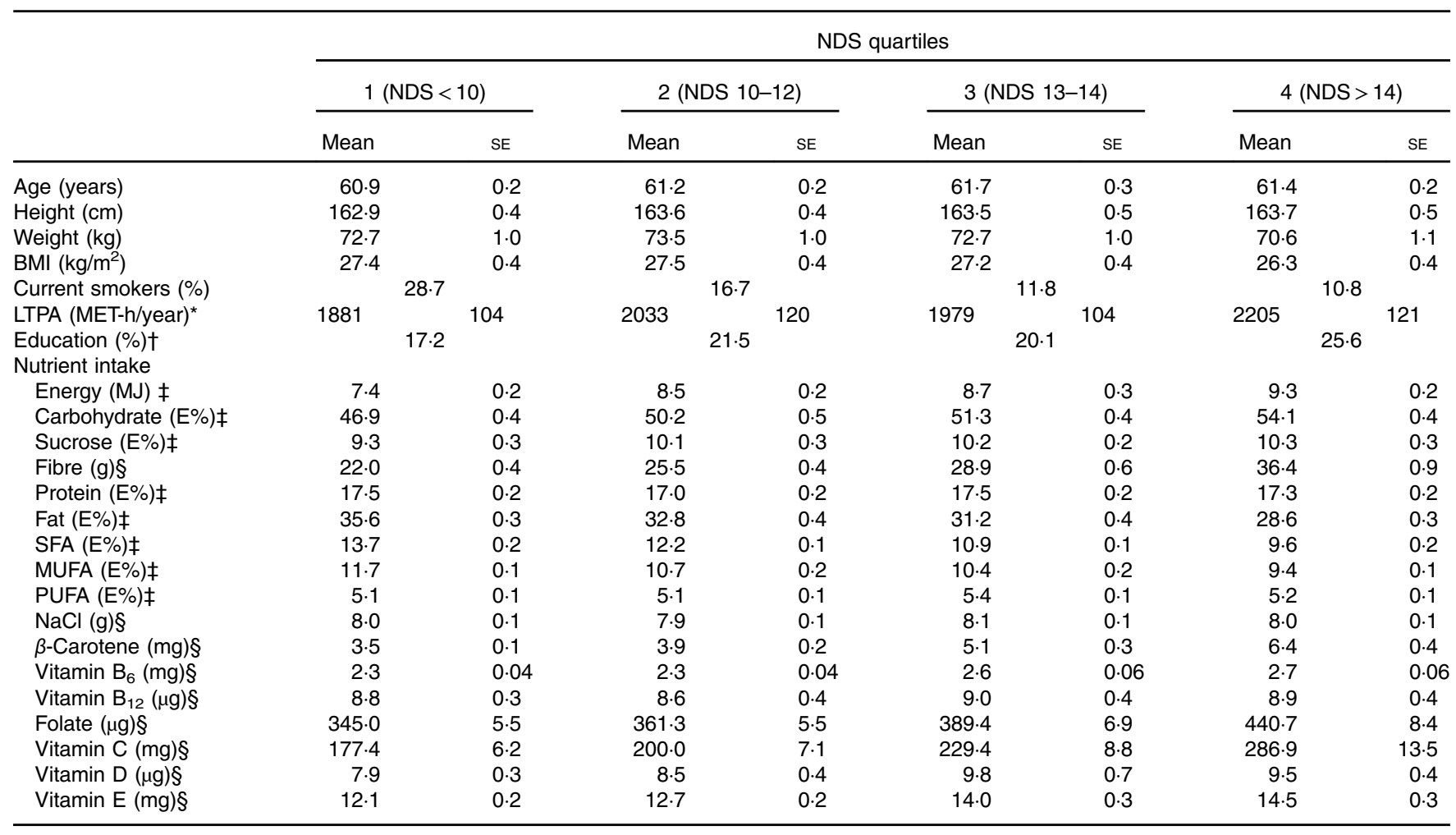

LTPA, leisure-time physical activity; MET, metabolic equivalent; E\%, percentage of total energy intake.

* 12-month history of LTPA.

$\dagger$ University degree.

$\ddagger$ Adjusted for age.

$\S$ Adjusted for age and daily energy intake.

arm curl and chair stand tests, reflecting better aerobic endurance and upper- and lower-body strength. Our results suggest that a healthy diet such as a healthy Nordic diet may help decrease the burden of functional limitations among older individuals.

Our study has important strengths including the use of a large study population consisting of both men and women. In addition, a long follow-up time provides an opportunity to explore the long-term influences of diet on physical performance. A further strength of our study is that we measured the overall physical performance instead of single aspects of it. SFT is validated and developed to measure especially older adults' physical capacity to perform normal everyday activities ${ }^{(31)}$. In contrast to the widely used SPPB, the SFT provides detailed information needed for evaluating specific physical functions such as aerobic endurance and flexibility. It has also been speculated ${ }^{(33)}$ that the SPPB contains items that have been found to be too easy (side-by-side balance test) or too difficult (five-times chair stand) to be effective performance discriminators for older, especially community-residing, people ${ }^{(1,34)}$.

There are some potential limitations that need to be considered when interpreting the results. First, as diet was assessed using an FFQ, participants might have overestimated the consumption of healthy food and under-reported the intake of food that is considered unhealthy ${ }^{(28)}$. However, the used FFQ was validated and it measures the entire diet and ranks subjects reasonably well according to their dietary intakes ${ }^{(27,28)}$. Second, even though we were able to adjust for a wide range of potential confounders including physical activity, we cannot exclude the possibility of residual confounding or effect modifications caused by other unmeasured covariates. In the present study, educational attainment was used as proxy of socio-economic status. We acknowledge that socio-economic status was higher among those with a high NDS than those with a lower score. In addition, individuals who had high NDS were more physically active than those with a low NDS. Therefore, even though the observed associations were robust to adjustment for socio-economic status and physical activity, future intervention studies may be particularly useful in determining whether a Nordic diet has also causal effects rather than serving as a marker of better overall quality of life. Third, individuals who participated in the follow-up clinical examination were younger, thinner, more educated and had a healthier diet at baseline compared with those who did not participate. Therefore, participants may not be fully representative of all older people living in Helsinki. Further, selective attrition over time may also introduce potential bias. These restrictions, however, rather underestimate than increase our ability to detect statistically significant associations between diet and overall physical performance. Finally, our study population came from an ethnically and culturally homogeneous Finnish population, which might limit the generalisability of our results to other populations or ethnicities. 
Table 2. Lifestyle factors and nutrient intakes according to the Nordic diet score (NDS) quartiles in men ( $n$ 472) at baseline (Mean values with their standard errors or proportions)

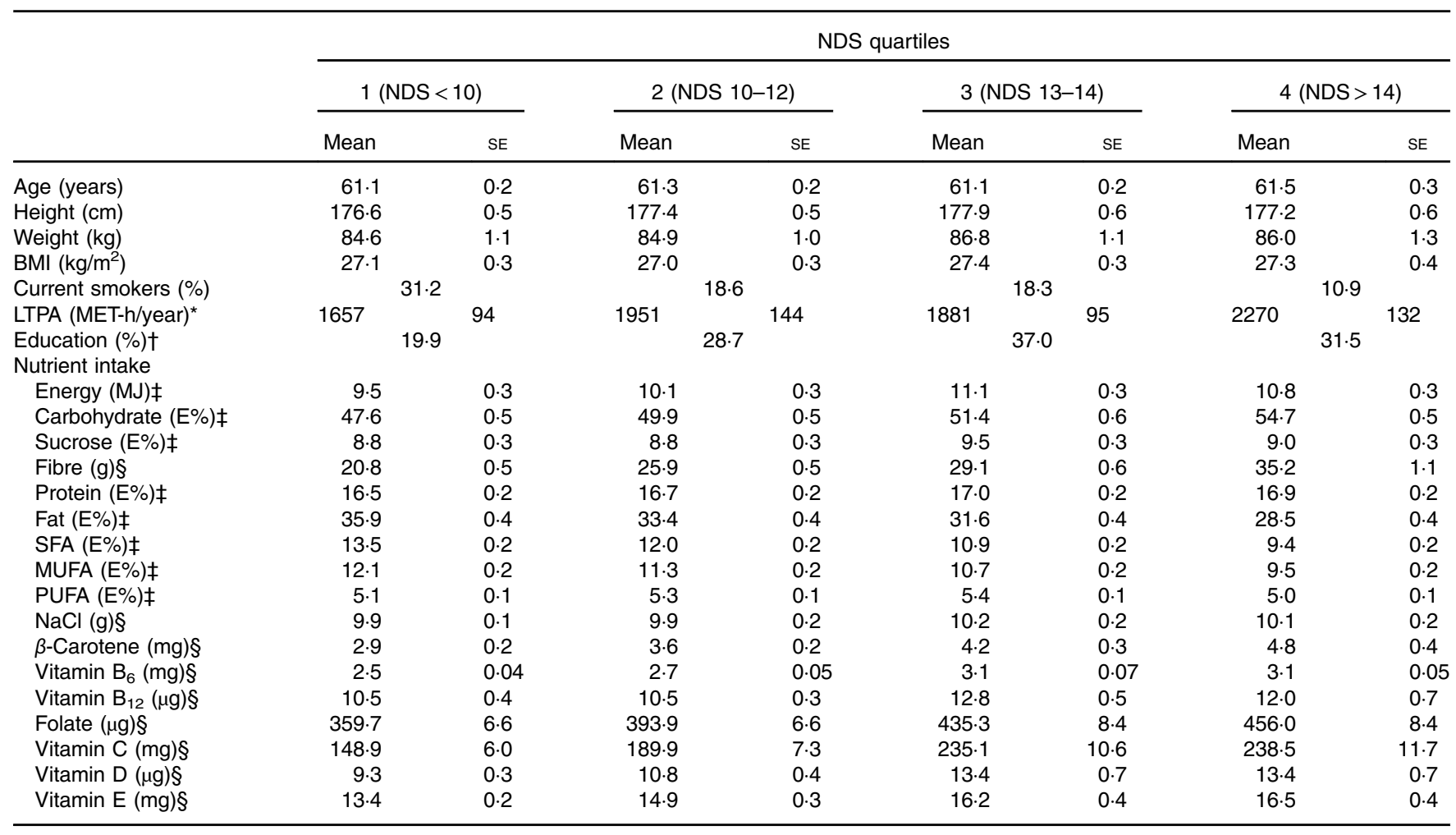

LTPA, leisure-time physical activity; MET, metabolic equivalent; E\%, percentage of total energy intake.

* 12-month history of LTPA.

$\dagger$ University degree.

$\ddagger$ Adjusted for age.

$\S$ Adjusted for age and daily energy intake.

To our knowledge, the present study is the first to focus on a healthy Nordic diet and its role on overall physical performance among older persons. However, previous studies have observed positive associations between the Mediterranean diet and adherence to nutrition recommendation and physical performance as well as frailty among older individuals ${ }^{(16-19,35)}$. A healthy Nordic diet resembles the Mediterranean diet, as the concept of the Mediterranean diet has been the inspiration for the concept of a healthy Nordic diet. Accordingly, both diets are rich in fruits, vegetables, whole grains, fish and low-fat dairy products. However, caution should be taken when comparing the results of a healthy Nordic diet with the Mediterranean diet as foods in these diets refer to different variants - for example, whole grains in the Mediterranean diet mostly come from dark wheat bread and pasta, whereas in a healthy Nordic diet whole grains mostly come from rye bread. However, both these diets show that a healthy diverse diet can be constructed from locally available foods. A healthy Nordic diet may be easier to implement in Nordic countries than a Mediterranean diet, as it takes into account local food resources and culture. It is known that local well-known foods that are part of the food culture are better adapted to the diet compared with foreign foods. In addition, a diet that contains locally available foods is also environmentally friendly.

We observed that a healthy Nordic diet was related to better overall physical performance in old women but not in men.
Similar sex differences were demonstrated by Martin et al. ${ }^{(36)}$ when they investigated the associations between single nutrients and foods and physical performance. There are several possible explanations for the observed sex differences. For example, our study included more women than men, which may limit the statistical power to detect significant associations among men. In addition, the life expectancy of women is longer than men, which could result in greater healthy survivor effects among men than women in our study population. Furthermore, studies have shown consistent sex-related behavioural differences in food choices - for example, it is known that men consume higher amounts of processed meat and lower amounts of fruits $^{(37,38)}$. Therefore, as the NDS was based on sex-specific quartiles, men with high NDS could consume lower amounts of favourable foods and higher amounts of unfavourable foods than women.

There are likely to be several mechanisms underlying the observed association. For example, several studies have shown that oxidative stress and inflammation may play a key role in the decrease of skeletal muscle strength and mass as well as overall physical performance, as proposed in a review by Semba et al. ${ }^{(39)}$. Previous epidemiological studies support this hypothesis by showing positive associations between antioxidants and physical performance ${ }^{(13,14)}$. In the present study, we observed that among those who had the highest 
Table 3. Participants' overall Senior Fitness Test (SFT) scores and points of SFT components according to the Nordic diet score (NDS) quartiles (Mean values with their standard errors; regression coefficients and $95 \%$ confidence intervals)

\begin{tabular}{|c|c|c|c|c|c|c|c|c|c|c|c|}
\hline & \multicolumn{8}{|c|}{ NDS quartiles } & \multirow[b]{3}{*}{ Regression coefficient ${ }^{\star}$} & \multirow[b]{3}{*}{$95 \% \mathrm{Cl}$} & \multirow[b]{3}{*}{$P \dagger$} \\
\hline & \multicolumn{2}{|c|}{1} & \multicolumn{2}{|c|}{2} & \multicolumn{2}{|c|}{3} & \multicolumn{2}{|c|}{4} & & & \\
\hline & Mean & $\mathrm{SE}$ & Mean & $\mathrm{SE}$ & Mean & SE & Mean & $\mathrm{SE}$ & & & \\
\hline \multicolumn{12}{|l|}{ Women } \\
\hline \multicolumn{12}{|l|}{ SFT score } \\
\hline Model $1 \ddagger$ & 43.4 & 1.3 & 45.5 & 1.4 & $48 \cdot 7$ & 1.4 & $51 \cdot 8$ & 1.7 & 0.81 & $0.48,1.15$ & $<0.001$ \\
\hline Model $2 \S$ & $45 \cdot 0$ & 1.2 & $45 \cdot 7$ & 1.2 & $48 \cdot 3$ & 1.2 & $49 \cdot 6$ & $1 \cdot 7$ & 0.55 & $0.22,0.88$ & 0.005 \\
\hline \multicolumn{12}{|l|}{ Chair stand } \\
\hline Model $1 \ddagger$ & 5.9 & 0.3 & $6 \cdot 2$ & 0.3 & $6 \cdot 6$ & 0.3 & 7.4 & 0.4 & 0.13 & $0.05,0.20$ & 0.001 \\
\hline Model $2 \S$ & $6 \cdot 1$ & 0.3 & $6 \cdot 2$ & 0.3 & 6.5 & 0.3 & $7 \cdot 3$ & 0.4 & 0.11 & $0.03,0.19$ & 0.006 \\
\hline \multicolumn{12}{|l|}{ 6-min walk } \\
\hline Model 1‡ & $9 \cdot 0$ & 0.4 & $10 \cdot 0$ & 0.4 & $10 \cdot 6$ & 0.4 & $12 \cdot 0$ & 0.5 & 0.26 & $0.16,0.36$ & $<0.001$ \\
\hline Model $2 \S$ & 9.6 & 0.3 & $10 \cdot 1$ & 0.4 & 10.5 & 0.4 & $11 \cdot 2$ & 0.5 & $0 \cdot 16$ & $0.06,0.26$ & 0.002 \\
\hline \multicolumn{12}{|l|}{ Arm curl } \\
\hline Model $1 \ddagger$ & $9 \cdot 1$ & 0.3 & $9 \cdot 9$ & 0.4 & $11 \cdot 1$ & 0.4 & $11 \cdot 3$ & 0.5 & 0.22 & $0.13,0.31$ & $<0.001$ \\
\hline Model $2 \S$ & 9.5 & 0.3 & $9 \cdot 9$ & 0.4 & $10 \cdot 9$ & 0.4 & 11.0 & 0.5 & 0.18 & $0.09,0.28$ & 0.001 \\
\hline \multicolumn{12}{|c|}{ Chair sit and reach } \\
\hline Model 1f & 9.7 & 0.4 & 9.5 & 0.5 & $9 \cdot 7$ & 0.4 & $10 \cdot 7$ & 0.6 & $0 \cdot 12$ & $0.01,0.23$ & 0.18 \\
\hline Model $2 \S$ & $10 \cdot 1$ & 0.4 & 9.5 & 0.5 & $9 \cdot 6$ & 0.4 & $10 \cdot 4$ & 0.6 & 0.06 & $-0.06,0.18$ & 0.68 \\
\hline \multicolumn{12}{|l|}{ Back scratch } \\
\hline Model 1f & 9.6 & 0.4 & $10 \cdot 0$ & 0.5 & $10 \cdot 8$ & 0.5 & $10 \cdot 5$ & 0.6 & $0 \cdot 10$ & $-0.01,0.21$ & 0.09 \\
\hline Model $2 \S$ & 9.9 & 0.4 & $10 \cdot 1$ & 0.4 & $10 \cdot 7$ & 0.4 & 9.9 & 0.5 & 0.04 & $-0.07,0.14$ & 0.58 \\
\hline \multicolumn{12}{|l|}{ Men } \\
\hline \multicolumn{12}{|l|}{ SFT score } \\
\hline Model $1 \ddagger$ & 41.8 & 1.3 & $42 \cdot 4$ & 1.6 & 43.4 & 1.6 & $42 \cdot 9$ & 1.6 & $0 \cdot 16$ & $-0.19,0.52$ & 0.51 \\
\hline Model $2 \S$ & $42 \cdot 7$ & 1.2 & $42 \cdot 4$ & 1.5 & $43 \cdot 2$ & 1.5 & $42 \cdot 0$ & 1.6 & -0.01 & $-0.36,0.35$ & 0.87 \\
\hline \multicolumn{12}{|l|}{ Chair stand } \\
\hline Model $1 \ddagger$ & 5.6 & 0.3 & $6 \cdot 0$ & 0.3 & $5 \cdot 8$ & 0.3 & 5.7 & 0.3 & 0.01 & $-0.07,0.07$ & 0.92 \\
\hline Model $2 \S$ & 5.8 & 0.3 & $6 \cdot 0$ & 0.3 & $5 \cdot 7$ & 0.3 & 5.5 & 0.3 & -0.03 & $-0.10,0.04$ & 0.43 \\
\hline \multicolumn{12}{|l|}{ 6-min walk } \\
\hline Model 1‡ & $10 \cdot 0$ & 0.5 & $10 \cdot 6$ & 0.5 & $10 \cdot 8$ & 0.5 & 11.6 & 0.6 & 0.14 & $0.02,0.26$ & 0.03 \\
\hline Model $2 \S$ & $10 \cdot 4$ & 0.4 & $10 \cdot 6$ & 0.4 & $10 \cdot 7$ & 0.5 & $11 \cdot 3$ & 0.5 & 0.08 & $-0.04,0.19$ & 0.19 \\
\hline \multicolumn{12}{|l|}{ Arm curl } \\
\hline Model $1 \ddagger$ & $8 \cdot 7$ & 0.4 & 9.4 & 0.5 & $10 \cdot 1$ & 0.4 & $9 \cdot 3$ & 0.5 & 0.09 & $-0.01,0.19$ & 0.17 \\
\hline Model $2 \S$ & 8.9 & 0.4 & 9.5 & 0.4 & $10 \cdot 0$ & 0.4 & $9 \cdot 0$ & 0.5 & 0.04 & $-0.07,0.15$ & 0.60 \\
\hline \multicolumn{12}{|c|}{ Chair sit and reach } \\
\hline Model 1f & 7.8 & 0.5 & 7.4 & 0.5 & 7.4 & 0.5 & $6 \cdot 9$ & 0.6 & -0.05 & $-0.17,0.07$ & 0.25 \\
\hline Model $2 \S$ & 7.8 & 0.5 & 7.4 & 0.5 & 7.4 & 0.5 & $6 \cdot 7$ & 0.6 & -0.08 & $-0.21,0.05$ & 0.13 \\
\hline \multicolumn{12}{|l|}{ Back scratch } \\
\hline Model 1‡ & $10 \cdot 0$ & 0.5 & $9 \cdot 0$ & 0.5 & 9.4 & 0.6 & $9 \cdot 6$ & 0.6 & -0.02 & $-0.15,0.10$ & 0.70 \\
\hline Model $2 \S$ & $10 \cdot 0$ & 0.4 & $8 \cdot 9$ & 0.5 & 9.5 & 0.6 & $9 \cdot 7$ & 0.6 & -0.01 & $-0.14,0.12$ & 0.82 \\
\hline
\end{tabular}

* Regression coefficient for change in SFT scores or points of SFT components for 1 point higher NDS.

$\dagger P_{\text {for a linear trend }}$ across fourths, tested by linear regression analyses.

$\ddagger$ Adjusted for age.

$\S$ Adjusted for age, energy intake, BMl, smoking status, educational attainment and physical activity.

score to a healthy Nordic diet had higher intake of several antioxidants including $\beta$-carotene and vitamins $\mathrm{C}$ and $\mathrm{E}$, compared with those with the lowest score. In addition, the dietary patterns associated significantly with better overall physical performance were higher intakes of fruits, berries and cereals, which all are linked with reduced oxidative stress and inflammation $^{(40)}$, and lower intakes of fat and red and processed meat. In contrast to fruits and cereals, intake of fat and red and processed meat have been shown to be related to increased oxidation as well as inflammation ${ }^{(40)}$. In addition, a healthy Nordic diet has been found to reduce the expressions of genes related to inflammation ${ }^{(23)}$ and to be associated with lower plasma levels of biomarkers of inflammation ${ }^{(41)}$. Therefore, we suggest that a healthy Nordic diet may prevent oxidative stress and inflammation, and thus have beneficial effects on overall physical performance.
We did not find significant associations between a healthy Nordic diet and upper- or lower-body flexibility, although flexibility is an important determinant of physical performance, and reduced flexibility can result in impaired mobility ${ }^{(42,43)}$. To our knowledge, previous studies have not investigated the association between the whole diet and flexibility in older adults. On the basis of our observations, we suggest that the role of diet on flexibility is minor, whereas other than dietary factors physical activity ${ }^{(44)}$ particularly plays a key role in determining flexibility.

From a public health point of view, our results are encouraging and introduce specific opportunities to promote physical performance in older age with better adherence to a healthy Nordic diet. It is known that even small improvements in physical performance have major impacts on a person's ability to live independently, and thus subsequently may decrease healthcare costs. In addition to 
Table 4. Participants' adjusted overall Senior Fitness Test score according to the Nordic diet score (NDS) component quartiles* (Mean values with their standard errors)

\begin{tabular}{|c|c|c|c|c|c|c|c|c|c|}
\hline & \multicolumn{9}{|c|}{ Quartile intakes of NDS components } \\
\hline & \multicolumn{2}{|c|}{1} & \multicolumn{2}{|c|}{2} & \multicolumn{2}{|c|}{3} & \multicolumn{2}{|c|}{4} & \multirow[b]{2}{*}{$P \dagger$} \\
\hline & Mean & $\mathrm{SE}$ & Mean & SE & Mean & SE & Mean & $\mathrm{SE}$ & \\
\hline \multicolumn{10}{|l|}{ Women } \\
\hline Cereals & $45 \cdot 1$ & $1 \cdot 3$ & 45.4 & 1.2 & 49.7 & $1 \cdot 3$ & 47.6 & 1.4 & 0.034 \\
\hline Vegetables & $45 \cdot 3$ & $1 \cdot 3$ & $48 \cdot 1$ & $1 \cdot 3$ & $48 \cdot 7$ & $1 \cdot 3$ & $45 \cdot 7$ & 1.4 & 0.73 \\
\hline Fruit and berries & $44 \cdot 0$ & $1 \cdot 2$ & $46 \cdot 4$ & 1.3 & $49 \cdot 8$ & $1 \cdot 3$ & $47 \cdot 6$ & 1.4 & 0.010 \\
\hline Low-fat milk & $45 \cdot 2$ & 1.4 & $46 \cdot 0$ & 1.3 & $49 \cdot 6$ & 1.2 & $46 \cdot 8$ & 1.3 & 0.15 \\
\hline Fish & $45 \cdot 9$ & 1.3 & 46.9 & 1.4 & $48 \cdot 3$ & 1.2 & $46 \cdot 5$ & 1.5 & 0.55 \\
\hline Fat ratio $\ddagger$ & $47 \cdot 3$ & 1.3 & $46 \cdot 3$ & 1.3 & $47 \cdot 0$ & 1.4 & $47 \cdot 1$ & 1.4 & 0.99 \\
\hline Fat $(E \%)$ & $49 \cdot 2$ & 1.4 & $47 \cdot 3$ & $1 \cdot 2$ & $47 \cdot 3$ & 1.2 & $44 \cdot 0$ & 1.4 & 0.006 \\
\hline Red and processed meat & $49 \cdot 7$ & 1.2 & 47.3 & 1.3 & $46 \cdot 0$ & 1.3 & $44 \cdot 7$ & 1.4 & 0.001 \\
\hline Alcohol§ & $46 \cdot 3$ & 0.7 & $50 \cdot 2$ & $1 \cdot 7$ & & & & & 0.029 \\
\hline \multicolumn{10}{|l|}{ Men } \\
\hline Cereals & 40.5 & 1.5 & $42 \cdot 3$ & 1.5 & 43.0 & 1.3 & 44.5 & 1.4 & 0.036 \\
\hline Vegetables & 43.5 & 1.4 & 42.5 & 1.4 & 41.5 & 1.6 & $42 \cdot 7$ & $1 \cdot 3$ & 0.57 \\
\hline Fruit and berries & $43 \cdot 0$ & 1.4 & 41.8 & 1.4 & $41 \cdot 7$ & 1.5 & $43 \cdot 8$ & 1.5 & 0.67 \\
\hline Low-fat milk & 44.5 & $1 \cdot 3$ & 44.4 & 1.7 & 41.4 & 1.4 & $40 \cdot 0$ & 1.4 & 0.009 \\
\hline Fish & 43.0 & 1.5 & 41.9 & 1.4 & $42 \cdot 3$ & 1.4 & $43 \cdot 1$ & 1.5 & 0.87 \\
\hline Fat ratioł & 44.3 & 1.4 & $42 \cdot 1$ & 1.5 & $41 \cdot 1$ & 1.4 & $42 \cdot 8$ & 1.5 & 0.40 \\
\hline Fat $(E \%)$ & $42 \cdot 7$ & 1.4 & $42 \cdot 8$ & 1.6 & $42 \cdot 4$ & 1.3 & $42 \cdot 4$ & 1.5 & 0.85 \\
\hline Red and processed meat & $44 \cdot 8$ & 1.5 & $42 \cdot 5$ & 1.4 & $40 \cdot 0$ & 1.4 & $43 \cdot 0$ & 1.5 & 0.16 \\
\hline Alcohol§ & $42 \cdot 6$ & 0.8 & 42.5 & 1.5 & & & & & 0.96 \\
\hline
\end{tabular}

$\mathrm{E} \%$, percentage for total energy intake.

* Adjusted for age, energy intake, BMI, smoking status, education and physical activity.

$\dagger P_{\text {for a linear trend }}$ across fourths, tested by linear regression analyses.

‡ Ratio of PUFA:SFA + trans-fatty acids.

$\S$ Women who consumed $<10 \mathrm{~g}$ of alcohol and men who consumed $<20 \mathrm{~g}$ of alcohol were in the lowest quartile.

physical performance, a healthy Nordic diet has other health benefits as it improves the blood lipid profile and insulin sensitivity, lowers blood pressure and inflammation and is linked with a smaller waist circumference ${ }^{(22,23,45,46)}$. Thus, a healthy Nordic diet seems to enhance physical performance as well as cardiovascular and overall health.

In conclusion, our study indicates that among women a healthy Nordic diet predicts better physical performance, and especially better aerobic endurance and upper- and lower-body strength 10 years later. In particular, high consumption of Nordic cereals and fruit and berries and low consumption of red and processed meat and total fat are related to better overall physical performance. Therefore, a healthy Nordic diet may subsequently decrease the risk of disability in old age. Future longitudinal studies are, however, needed to determine whether the beneficial associations of a healthy Nordic diet on physical performance are also found in other Nordic countries and closer regions.

\section{Acknowledgements}

The authors thank the volunteers for taking the time to participate in the clinical study, the research nurses for carrying out the clinical examinations and Niina Kaartinen, Tommi Korhonen and Sigrid Rosten for assisting with the technical aspects of the study.

The present study was financially supported by grants from the Academy of Finland, Finska Läkaresällskapet, Samfundet Folkhälsan, Juho Vainio Foundation, Signe and Ane Gyllenberg Foundation, Yrjö Jahnsson Foundation, Diabetes Research Foundation, Finnish Foundation for Cardiovascular Research and Emil Aaltonen Foundation. The funding sources had no role in the study design; collection, analysis or interpretation of the data; or writing of the report.

The contribution of each author was as follows: M.-M. P. wrote the first draft of the manuscript and carried out the statistical analysis; M.-M. P., M. V. B. and J. G. E. contributed to the conception and design of the study; M. K. S., N. K., M. S., S. M. and E. K. participated in the collection and preparation of the data; M. V. B., P. P. and T. R. participated in the conception of the physical performance test. All the authors contributed to the critical revision of the manuscript.

None of the authors has any conflicts of interest to declare.

\section{References}

1. Guralnik JM, Simonsick EM, Ferrucci L, et al. (1994) A short physical performance battery assessing lower extremity function: association with self-reported disability and prediction of mortality and nursing home admission. J Gerontol 49 , M85-M94.

2. Cooper R, Kuh D, Hardy R, et al. (2010) Objectively measured physical capability levels and mortality: systematic review and meta-analysis. BMJ 341, c4467.

3. Cooper R, Kuh D, Cooper C, et al. (2011) Objective measures of physical capability and subsequent health: a systematic review. Age Ageing 40, 14-23.

4. Legrand D, Vaes B, Mathei C, et al. (2014) Muscle strength and physical performance as predictors of mortality, hospitalization, and disability in the oldest old. J Am Geriatr $S o c \mathbf{6 2}$, $1030-1038$. 
5. Onder G, Penninx BW, Ferrucci L, et al. (2005) Measures of physical performance and risk for progressive and catastrophic disability: results from the Women's Health and Aging Study. J Gerontol A Biol Sci Med Sci 60, 74-79.

6. den Ouden ME, Schuurmans MJ, Arts IE, et al. (2013) Association between physical performance characteristics and independence in activities of daily living in middle-aged and elderly men. Geriatr Gerontol Int 13, 274-280.

7. von Bonsdorff M, Rantanen T, Laukkanen P, et al. (2006) Mobility limitations and cognitive deficits as predictors of institutionalization among community-dwelling older people. Gerontology 52, 359-365.

8. World Health Organization (2011) Global Health and Ageing. http://www.who.int/ageing/publications/global_health/en/ (accessed July 2015).

9. Katz S, Branch LG, Branson MH, et al. (1983) Active life expectancy. $N$ Engl J Med 309, 1218-1224.

10. Houston DK, Cesari M, Ferrucci L, et al. (2007) Association between vitamin $\mathrm{D}$ status and physical performance: the InCHIANTI study. J Gerontol A Biol Sci Med Sci 62, 440-446.

11. Tieland M, Brouwer-Brolsma EM, Nienaber-Rousseau C, et al. (2013) Low vitamin D status is associated with reduced muscle mass and impaired physical performance in frail elderly people. Eur J Clin Nutr 67, 1050-1055.

12. Wicherts IS, van Schoor NM, Boeke AJ, et al. (2007) Vitamin D status predicts physical performance and its decline in older persons. J Clin Endocrinol Metab 92, 2058-2065.

13. Bartali B, Frongillo EA, Guralnik JM, et al. (2008) Serum micronutrient concentrations and decline in physical function among older persons. JAMA 299, 308-315.

14. Semba RD, Varadhan R, Bartali B, et al. (2007) Low serum carotenoids and development of severe walking disability among older women living in the community: the women's health and aging study I. Age Ageing 36, 62-67.

15. Rousseau JH, Kleppinger A \& Kenny AM (2009) Self-reported dietary intake of omega-3 fatty acids and association with bone and lower extremity function. J Am Geriatr Soc 57, 1781-1788.

16. Zbeida M, Goldsmith R, Shimony T, et al. (2014) Mediterranean diet and functional indicators among older adults in nonMediterranean and Mediterranean countries. J Nutr Health Aging 18, 411-418.

17. Milaneschi Y, Bandinelli S, Corsi AM, et al. (2011) Mediterranean diet and mobility decline in older persons. Exp Gerontol 46, 303-308.

18. Shahar DR, Houston DK, Hue TF, et al. (2012) Adherence to Mediterranean diet and decline in walking speed over 8 years in community-dwelling older adults. J Am Geriatr Soc 60, $1881-1888$.

19. Xu B, Houston DK, Locher JL, et al. (2012) Higher Healthy Eating Index-2005 scores are associated with better physical performance. J Gerontol A Biol Sci Med Sci 67, 93-99.

20. Adamsson V, Reumark A, Cederholm T, et al. (2012) What is a healthy Nordic diet? Foods and nutrients in the NORDIET study. Food Nutr Res 56, 10.3402/fnr.v56i0.18189.

21. Kanerva N, Kaartinen NE, Schwab U, et al. (2013) The Baltic Sea Diet Score: a tool for assessing healthy eating in Nordic countries. Public Health Nutr 17, 1697-1705.

22. Adamsson V, Reumark A, Fredriksson IB, et al. (2011) Effects of a healthy Nordic diet on cardiovascular risk factors in hypercholesterolaemic subjects: a randomized controlled trial (NORDIET). J Intern Med 269, 150-159.

23. Kolehmainen M, Ulven SM, Paananen J, et al. (2015) Healthy Nordic diet downregulates the expression of genes involved in inflammation in subcutaneous adipose tissue in individuals with features of the metabolic syndrome. Am J Clin Nutr 101, 228-239.
24. Olsen A, Egeberg R, Halkjær J, et al. (2011) Healthy aspects of the Nordic diet are related to lower total mortality. J Nutr $\mathbf{1 4 1}$, 639-644.

25. Barker DJ, Osmond C, Forsén TJ, et al. (2005) Trajectories of growth among children who have coronary events as adults. N Engl J Med 353, 1802-1809.

26. Ylihärsilä H, Kajantie E, Osmond C, et al. (2008) Body mass index during childhood and adult body composition in men and women aged 56-70 y. Am J Clin Nutr 87, $1769-1775$

27. Männistö S, Virtanen M, Mikkonen T, et al. (1996) Reproducibility and validity of a food frequency questionnaire in a case-control study on breast cancer. J Clin Epidemiol 49, 401-409.

28. Paalanen L, Männistö S, Virtanen MJ, et al. (2006) Validity of a food frequency questionnaire varied by age and body mass index. J Clin Epidemiol 59, 994-1001.

29. Reinivuo H, Hirvonen T, Ovaskainen ML, et al. (2010) Dietary survey methodology of FINDIET 2007 with a risk assessment perspective. Public Health Nutr 13, 915-919.

30. Rikli RE \& Jones CJ (1999) Development and validation of a functional fitness test for community-residing older adults. J Aging Phys Act 6, 127-159.

31. Rikli RE \& Jones CJ (2013) Senior Fitness Test Manual, 2nd ed. Champaign, IL: Human Kinetics.

32. Lakka TA \& Salonen JT (1992) Intra-person variability of various physical activity assessments in the Kuopio Ischaemic Heart Disease Risk Factor Study. Intern J Epidemiol 21, 467-472.

33. Rikli RE \& Jones CJ (2013) Development and validation of criterion-referenced clinically relevant fitness standards for maintaining physical independence in later years. Gerontologist 53, 255-267.

34. Seeman TE, Charpentier PA, Berkman LF, et al. (1994) Predicting changes in physical performance in a high-functioning elderly cohort: MacArthur studies of successful aging. J Gerontol 49, M97-108.

35. León-Muñoz LM, Guallar-Castillón P, López-García E, et al. (2014) Mediterranean diet and risk of frailty in community-dwelling older adults. J Am Med Dir Assoc 15, 899-903.

36. Martin H, Aihie Sayer A, Jameson K, et al. (2011) Does diet influence physical performance in community-dwelling older people? Findings from the Hertfordshire Cohort Study. Age Ageing 40, 181-186.

37. O'Doherty Jensen K \& Holm L (1999) Preferences, quantities and concerns: socio-cultural perspectives on the gendered consumption of foods. Eur J Clin Nutr $\mathbf{5 3}$, 351-359.

38. Ovaskainen ML, Paturi M, Tapanainen H, et al. (2010) Educational differences in the diet of Finnish adults and the associations between education and the determinants and facilitators of dietary fat quality. Public Health Nutr 13, 925-931.

39. Semba RD, Lauretani F \& Ferrucci L (2007) Carotenoids as protection against sarcopenia in older adults. Arch Biochem Biophys 458, 141-145.

40. Calder PC, Ahluwalia N, Brouns F, et al. (2011) Dietary factors and low-grade inflammation in relation to overweight and obesity. Br J Nutr 106, Suppl. 3, S5-S78.

41. Kanerva N, Loo BM, Eriksson JG, et al. (2014) Associations of the Baltic Sea diet with obesity-related markers of inflammation. Ann Med 46, 90-96.

42. Holland GJ, Tanaka K, Shigematsu R, et al. (2002) Flexibility and physical functions of older adults: a review. J Aging Phys Act 10, 169-206. 
43. Nelson ME, Rejeski WJ, Blair SN, et al. (2007) Physical activity and public health in older adults: recommendation from the American College of Sports Medicine and the American Heart Association. Med Sci Sports Exerc 39, 1435-1445.

44. Simons R \& Andel R (2006) The effects of resistance training and walking on functional fitness in advanced old age. J Aging Health 18, 91-105.
45. Kanerva N, Kaartinen NE, Schwab U, et al. (2013) Adherence to the Baltic Sea diet consumed in the Nordic countries is associated with lower abdominal obesity. Br J Nutr 109, 520-528.

46. Uusitupa M, Hermansen K, Savolainen MJ, et al. (2013) Effects of an isocaloric healthy Nordic diet on insulin sensitivity, lipid profile and inflammation markers in metabolic syndrome-a randomized study (SYSDIET). J Intern Med 274, 52-66. 